\title{
Swimming and bone: Is low bone mass due to hypogravity alone or does other physical activity
}

influence it?

Gómez-Bruton A, MSc ${ }^{1,2}$; González-Agüero A, $\mathrm{PhD}^{1,3}$; Gómez-Cabello A, $\mathrm{PhD}^{1,4}$; Matute-Llorente A,

$\mathrm{MSc}^{1,2}$; Casajús JA, MD, PhD ${ }^{1,2 ;}$ Vicente-Rodríguez G, $\mathrm{PhD}^{1,2}$.

${ }^{1}$ GENUD “Growth, Exercise, NUtrition and Development” Research Group, University of Zaragoza,

Zaragoza, Spain.

${ }^{2}$ Faculty of Health and Sport Sciences (FCSD), Department of Physiatry and Nursing, University of

Zaragoza, Ronda Misericordia 5, 22001-Huesca, Spain.

${ }^{3}$ Department of Sport and Exercise Science, Aberystwyth University, Ceredigion, United Kingdom.

${ }^{4}$ Centro Universitario de la Defensa, Zaragoza, Spain

Running head: Swimming, bone and weight-bearing activity

\section{Corresponding author:}

German Vicente-Rodriguez, PhD.

GENUD (Growth, Exercise, NUtrition and Development) Research Group

Faculty of Health and Sport Sciences

University of Zaragoza

Grupo GENUD Edificio SAI $2^{\mathrm{a}}$ planta, Zaragoza

Phone: +34 876553754 or 976761000 (Ext. 843754)

Fax: +34 974238422 / +34 974239384

Email: gervicen@unizar.es

\section{ACKNOWLEDGEMENTS}

We would like to thank participants and their families and coaches for their collaboration. Special thanks are given to Kenn Konstabell from the National Institute for Health Development of Estonia for his help with the statistical analyses and to Lindsey A. Bruton from the University San Jorge of Spain for her work in reviewing the English style and grammar. This work was supported by the Spanish 'Ministerio de Economia y competitividad' 'Plan Nacional I+D+i 2008-2011 (Project DEP2011-29093)'. This project has been co-financed by "Fondo Europeo de Desarrollo Regional” (MICINN-FEDER). AGB received a Grant FPI 2012 (BES-2012-051888) from the 'Ministerio Economía y Competitividad'. AML received a Grant AP12-02854 from the ‘Ministerio de Educación Cultura y Deportes’. Alejandro Gomez-Bruton, Alejandro Gonzalez-Agüero, Alba Gomez-Cabello, Angel Matute-Llorente, Jose Antonio Casajús, and Germán Vicente-Rodríguez declare that they have no conflicts of interest. 


\section{ABSTRACT}

Swimming during adolescence has shown neutral or even negative effects on bone mass. Nevertheless, it is still unknown if these effects are due to swimming or to other factors, such as sedentary behaviors.

Purpose: Three objectives were described; 1 ) to measure objective physical activity (PA) additional to swimming performed by adolescent swimmers (SWI) and compare it to that performed by normo-active controls (CG), 2) to describe the relationship between objectively measured PA and bone mass, and 3) to compare bone mass of swimmers that meet the World Health Organization PA guidelines (active) WHO and those that do not (inactive).

Methods: A total of 71 SWI (33 Females) and 41 CG (17 females) wore an accelerometer for at least 4 days. PA was expressed as the amount of time (minutes/day) in each intensity [sedentary/light/moderate or vigorous (VPA), and the sum of moderate and vigorous (MVPA)]. Using the cut-off points proposed by Vanhelst et al. SWI were classified as active or inactive according to whether they reached 60 minutes of weight-bearing MVPA per day or not. Bone mineral density (BMD) was measured by dual energy Xray absorptiometry, and bone strength values were calculated with peripheral quantitative computed tomography. Differences in PA intensities were calculated between SWI and CG. The relation of VPA to bone mass was studied in the SWI.

Results: Male-SWI spend lower amount of time in VPA and MVPA than male-GC, was associated with lower BMD values in SWI than CG.

Conclusion: Swimming may displace weight bearing VPA with serious implications on bone health.

Keywords: Swimming, bone mass, accelerometry, pQCT, DXA 


\section{INTRODUCTION}

Physical activity (PA), specifically participation in sport during growth, seems to be effective in reducing the prevalence of osteoporosis-related fractures[1], which are estimated to have a mortality prevalence of up to $39 \%$ in women and 51\% in men aged over 60 years[2]. Although osteoporotic fractures usually occur in the elderly, the idea that "senile osteoporosis is a paediatric disease"[3] is increasingly accepted[4]. Therefore, prevention has become one of the most important tools to fight this disease[5], with sport participation being highly recommended, particularly during childhood[6]. However, not all sports have the same effect on bone mass[6, 7].

High impact sports seem to be more beneficial to bone mass than non-impact sports such as cycling[8] or swimming[9]. A recent systematic review[9] showed that swimming did not have a positive effect on bone mass. However, due to differences in the results between studies it is not possible to assert that swimming was actually negative to bone mass acquisition. A further meta-analyses focusing on bone mineral density (BMD) of the lumbar spine, femoral neck and whole body showed that adolescent swimmers (SWI) presented similar BMD values when compared to sedentary controls and lower values when compared to other athletes[10].

A recent study showed that adolescent SWI presented lower values of BMD than sedentary controls at several sites when measured by dual-energy X-ray absorptiometry (DXA)[11] and similar values for the forearm and tibia for bone strength indices in evaluations performed by peripheral quantitative computerized tomography (pQCT)[12]. This can be explained by the fact that the forearm, particularly the radius, undergoes a high degree of strain during swimming.

The lower BMD found in adolescent SWI when compared to normo-active controls evaluated with DXA are alarming, as adolescence is a key stage for attaining peak bone mass in which a 5 to $10 \%$ change may result in a $25-50 \%$ difference in hip fracture later in life[13]. Therefore, the etiology of these differences should be carefully studied.

Two hypotheses emerge when trying to explain these differences. The first is environment-based, as SWI spend many hours in water, a medium with low gravity. The effects of hypogravity have been well described in astronauts[14] who, like SWI, operate in a low-gravity environment, with minimal impact on bone mass. Therefore, only relatively low tensions are transmitted by the muscular system to the bone during this type of exercise. Similarly, many studies have been developed in swimmers finding similar or lower BMD values than sedentary controls and similar bone strength indices (For review[9])

The second hypothesis is behavioral. Adolescent SWI spend several hours in water exercising. These daily hours could displace other weight-bearing physical activities that have been shown to be more advantageous to bone mass than swimming in more than 15 studies (for review[9]). In addition, adolescent swimmers have to comply with school-tasks, rest to recover from swimming and sleep. This would result in no PA nor impact at all, increasing the number of sedentary hours and reducing the time spent in weight-bearing moderate or vigorous activities.

To date, no studies have been performed evaluating PA levels in adolescent SWI comparing them to a control group of normo-active counterparts (CG). Therefore, it remains unknown if the lower BMD levels reported in swimmers[11] are due to the swimming per se or if, in fact, their sedentary levels are greater than those of their CG.

Therefore, the aims of this study were; 1 ) to evaluate and compare free-living PA performed by adolescent SWI and normo-active controls, 2) to describe the associations between weight-bearing PA and bone mass in SWI, and 3)to compare bone mass between swimmers that meet the PA guidelines (active) and those that do not meet the PA guidelines (inactive).

\section{METHODS}

\section{Participants}

Of the 235 informed consent forms delivered, a total of 173 were returned signed and agreed to participate in the study (98 SWI and 75 controls). However, those not meeting the following inclusion criteria were excluded a posteriori: being between the ages of 11 and 18, Caucasian, healthy, non-smoker, 
with no diagnosed chronic disease or musculoskeletal disorders (fibromyalgia, gout, osteoarthritis, rheumatoid arthritis, tendinitis), bone fractures or medication. SWI had a history of swimming and competing in regional tournaments for more than 3 years and trained for a minimum of 6 hours per week, while normo-active controls (CG) were not engaged in any aquatic activity on a regular basis or practicing any sport for more than 3 hours per week.

A total of 77 SWI (34 girls) and 52 CG (23 girls) met the previous inclusion criteria and participated in the study. SWI were recruited from 4 swimming clubs from the city of Zaragoza (Spain), while participants in the CG were recruited from 3 high-schools in the same city.

Subjects were asked to answer a medical questionnaire and parents gave additional information regarding medical information such as past injuries, medication and known diseases.

\section{Ethics statement.}

Written informed consent was obtained from parents and adolescents. The study was performed following the ethical guidelines of the Declaration of Helsinki 1961 (revised in Fortaleza, 2013). The study protocol was approved by the Ethics Committee of Clinical Research from the Government of Aragón (ref. CP08/2012, CEICA, Spain).

\section{Experimental design}

The data presented herein corresponds to a cross-sectional study within a larger randomized controlled trial[15] that has been registered in a public database (ClinicalTrials.gov identifier [NCT02380664]).

All participants were asked to visit the laboratory for a period of 3 hours to complete the evaluations that took place between September and December 2012. All the tests and questionnaires were performed by qualified researchers from the University of Zaragoza and are explained elsewhere[15]. Radiation exposure to adolescent athletes was limited and approved by the Spanish Nuclear Security Agency.

\section{Anthropometric measures}

Height was measured with a stadiometer without shoes and minimal clothing to the nearest $0.1 \mathrm{~cm}$ (SECA 225, SECA, Hamburg, Germany) and weight to the nearest $0.1 \mathrm{~kg}$ (SECA 861, SECA, Hamburg, Germany). Body mass index (BMI) was calculated as weight (in kilograms) divided by height squared (in meters).

\section{Pubertal status assessment}

Pubertal maturation was determined by self-assessment of secondary sexual characteristics according to the criteria devised by Tanner[16]. This method has been reported to be both valid and reliable in assessing sexual maturity among adolescent athletes[17].

\section{Bone, lean and fat mass assessed by Dual Energy-X ray absorptiometry}

Scanning procedures and specific results are detailed elsewhere[11]. Scans for the whole body, lumbar spine, and non-dominant forearm and femoral neck were performed with DXA using a pediatric version of the software QDR-Explorer (QDR-Explorer, Hologic Corp., Software version 12.4, Bedford, MA, USA). For the present study the trochanter, femoral neck, pelvis, lumbar spine, arms, legs, radius and subtotal body BMD were used.

\section{Bone structure and strength assessed by peripheral quantitative computed tomography}

Scanning procedures and specific results are detailed elsewhere[12, 18]. The non-dominant radius and tibia were measured with a Stratec XCT-2000 L scanner (Stratec Medizintechnik, Pforzheim, Germany). Both regions were measured at metaphyseal and diaphesal sites. For the present study cortical thickness (CRT_THCK) was measured, and fracture load on the $\mathrm{X}$ axis (FRAC_X), polar strength strain index (SSIPOL) and bone strength index (BSI) were calculated.

\section{Physical activity assessment}

Free-living PA levels and patterns were objectively assessed using the uniaxial Actitrainer accelerometer (ActiGraph, LLC, Pensacola, FL, USA). The accelerometer records continuously and has no threshold for 
activity intensity. It was set to record PA in a 15-s epoch on the basis of literature related to sudden bursts of activity common to youth[19].

Subjects wore the accelerometer for 6 consecutive days (including at least 1 weekend day). Participants were instructed to wear the accelerometer on their lower back using an elastic belt, adjusted to ensure close contact with the body. The subjects were required to wear the accelerometers from the moment they woke up in the morning until bed time. The only moment of the day that accelerometers could be removed was when participants were going to perform aquatic activities or while bathing or showering due to the fact that these accelerometers are not waterproof. In addition, participants took off their accelerometer when they went to bed and put them back on again first thing in the morning.

\section{Data reduction}

Accelerometer data were analyzed using algorithms developed in the software R (www.rproject.org). A set of add-on functions to $\mathrm{R}$ were developed to allow $\mathrm{R}$ to automatically read in the accelerometer raw files; edit the data to exclude the likely non-wearing periods and compute daily summary statics. Two rules were used for excluding data: 1) all negative counts were replaced by missing data code, and 2) periods of 20 minutes or more consecutive zero counts were replaced by missing data code before further analysis as previous studies showed that so many consecutive zeroes are not observed in an awake child wearing and accelerometer and thus is inconsistent with monitor wear[20]. The output generated by $\mathrm{R}$ included accelerometer average counts per minute (CPM), sedentary time and physical activities of different intensities based on Vanhelst cut offpoints[21].

Minutes of valid time in sedentary, light (LPA), moderate (MPA), vigorous (VPA), and the sum of moderate to vigorous PA (MVPA) intensities were calculated from the raw data obtained from the accelerometer using thresholds validated against spyro-ergometry and heart rate monitoring in adolescents that were of a similar age to our sample[21]. Based on these cut off points the activity categories and accelerometer counts were; sedentary activity, 0-400 CPM; light activity, 401-1900 CPM; moderate activity, 1901-3918 CPM; and vigorous activity, greater than 3918 CPM, respectively. In the present study, total minutes of daily physical activity is equivalent to valid time which is the average daily time spent wearing the accelerometer once the data reduction was performed according to Ojiambo et al.[22]. The final criteria for including the data in the statistical analysis was that the accelerometer had registered for at least 4 days (including one weekend day) and for at least 8 hours each day.

\section{Statistical analysis}

All the analyses were performed using the statistical package SPSS 15.0 for Windows (SPSS, Inc, Chicago, IL), and significance was set at $\mathrm{p}<0.05$. All the analyses were performed for the whole sample and stratified by gender. Mean and standard deviation are given as descriptive statistics. Independent $t-$ tests were performed to compare descriptive characteristics between SWI and CG. A Chi-square test was performed to evaluate differences in Tanner stage between SWI and CG. Analyses of covariance (ANCOVA) were performed to evaluate differences between SWI and CG in daily minutes in different PA intensities, with valid time as a covariate and Bonferroni corrections.

In addition, gender stratified multiple linear regression models adjusted for age and lean mass were constructed to examine the effects of MVPA on bone evaluated by DXA and pQCT. We tested for group interactions (SWI vs CG), in order to describe whether VPA influenced bone differently in SWI and CG.

The previous multiple linear regressions were repeated without the interaction term exclusively in the SWI in order to describe the effect of weight-bearing activity on SWI.

Finally, SWI were classified as: active or inactive if they complied or not with the PA guidelines[23] (60 minutes of MVPA). Age, subtotal lean and height or radius/tibia length (for DXA or pQCT variable respectively) ANCOVAs were performed to evaluate differences in bone mass between those active and inactive SWI with Bonferroni corrections by the number of bone parameters. In addition, a Chi-square test was performed to determine if differences existed between SWI and CG regarding compliance of PA guidelines.

\section{RESULTS}




\section{Participants}

Final reduction of accelerometers data resulted in the loss of 17 participants (6 SWI and $11 \mathrm{CG}$ ). Therefore, the final sample consisted of 70 SWI (33 Females) and 41 CG (17 females).

Age and physical characteristics are summarized in Table 1. As a whole sample, SWI were taller, heavier, and presented higher lean mass than CG, with these differences present in males but not in females when viewed by gender (all $\mathrm{p}<0.05$; table 1 ). In addition, swimmers presented a higher maturation status than CG ( $<<0.05$; table 1$)$, although these differences disappeared when viewed by gender.

\section{Physical activity}

Total minutes in each PA intensity adjusted by valid time are presented in Table 2.

SWI as a whole group presented lower amounts of time spent in VPA and MVPA than CG (both power:0.577 and 0.567 respectively, both $\mathrm{p}<0.05$; table 2 ) however, when stratified by gender, only male SWI presented lower VPA and MVPA spent time than male-CG (power:0.711 and 0.553 respectively, both $\mathrm{p}<0.05$; table 2).

\section{Group interactions}

No group interactions were found for the females. However, for males, radius CRT_THCK, radius BSI, tibia SSIPOL and tibia FRAC_X, presented positive group by PA interaction (all $\mathrm{p}<0.05$ ), suggesting that weight-bearing PA influenced SWI bone more than CG bone.

\section{Swimmers and VPA}

When studying the effect of VPA on bone in SWI, no relationship was found between VPA and BMD at any of the studied variables (all p>0.05; data not shown). However, for the male group, VPA explained radius CRT_THCK and tibia FRAC_X axis, both calculated with pQCT (all p<0.05; Table 3).

\section{Active Vs. Inactive swimmers}

As no differences were found between female active and inactive SWI, only differences between active and inactive male SWI are plotted in Figure 1.

Male SWI subtotal body, arms, legs and radius BMD values were higher in active than in inactive SWI (all $\mathrm{p}<0.05$; figure 1A). In addition, active SWI presented higher tibia SSIPOL, FRAC_X and BSI when compared to inactive SWI (all $\mathrm{p}<0.05$; figure $1 \mathrm{~B}$ ). For the radius, BSI and CRT_THCK were also higher in active SWI than in inactive SWI (both $\mathrm{p}<0.05$; figure $1 \mathrm{C}$ and $1 \mathrm{D}$ respectively).

\section{DISCUSSION}

The main finding of the present study is that, without taking into account swimming time, adolescent male SWI present different PA patterns when compared to controls which appear to influence their bone mass acquisition.

Contrary to our initial hypothesis, SWI did not present more sedentary time than CG. Sedentary behaviors have shown to be negative to bone mass in several studies[24, 25]. Therefore, the lower BMD values presented in our sample of SWI when compared to CG in a previous study[11] and in studies developed by other researchers[26-28] should not be attributed to longer periods of time spent in sedentary behaviors.

On the other hand, PA levels (MVPA and VPA) were higher in CG than in SWI, although when stratified by gender, differences were only significant in the male group. All the statistical analyses evaluating the relation between bone and PA were performed with VPA as it has been shown to be a better predictor of bone mass than MVPA[29] and other intensities[30, 31].

The positive interactions between groups (SWI and CG), VPA, BMD and several strength indices deserve special attention, as they suggest that VPA affects bone health more in SWI than in CG. This could be related to Frost's mechanostat theory[32] which indicated that a minimum effective strain for modeling the bone (2000 microstrains) was needed. A recent review performed by Skerry et al. [33] indicated that 
these thresholds may be relative to the individual's habitual loads, and thus if SWI are performing continuous low loads it is possible that their bone remodeling threshold may be lower than the norm. Therefore, less vigorous activity might have a greater effect on swimmers' bone.

The effects of PA on health outcomes like bone mass are clear, although the volume thresholds and intensities to obtain these benefits still remain unclear for different populations. As stated by Janssen et al.[34] it is unknown as to whether a child who performs a daily hour of PA on a regular basis would have any greater health benefits than a child that accumulates 7 hours of activity over the week, with different amounts of activity being performed each day (including some days with no activity).

In addition to the previous interactions, when focusing exclusively on SWI, VPA predicted several bone mass variables, even when taking age and lean mass into account. Although these predictive values were not very high, they showed improvements in the prediction of the tibia resistance to fracture and radius cortical thickness (Change in $\mathrm{r}^{2}=0.05$ and 0.09 respectively), suggesting that performing an extra activity in addition to swimming did indeed improve their bone mass. This is of critical relevance for the literature focusing on this topic, as there are inconsistent results regarding SWI BMD when compared to CG (for complete review[9]). These findings are in line with previous reports suggesting that practicing other sports in addition to swimming might improve bone mass [11, 35]. Therefore, further studies focusing on bone mass in SWI should make the effort to divide SWI into pure SWI (those who only practice swimming) and non-pure SWI (SWI who do swimming and other physical activities), as mixing both groups may mask results.

The positive influence of VPA on bone was accompanied by positive effects of MVPA on bone, as those SWI that did comply with the 60 minutes of daily recommend MVPA presented higher BMD evaluated by DXA, and radius and tibia PQCT values than SWI who did not reach the 60 minutes of MVPA. Several studies have defined the positive effects of weight-bearing activities on bone in adults[7] and during growth[6]. Therefore, these results were expected and reinforce the importance that impact activities have on bone development.

Many SWI did not attain the recommended amount of MVPA as registered by the accelerometers. However, this is not worrying in terms of cardiovascular health, as they are meeting these [23] with swimming (which was not registered by the accelerometers, but assistance to sessions was registered by coaches with an average training of 10 hours per week). The prevalence of CG reaching MVPA (68\% for the boys and $47 \%$ for the girls), was slightly higher than previous reports that measured European adolescents (57\% boys and 28\% girls)[36]. This higher prevalence for both groups (CG and SWI) could partly be explained by the mandatory classes of PA that they have to perform during the week at school, an issue that was previously addressed[11], and that could have made the CG more active than others from previous studies (although the SWI might also be stronger than SWI from other samples). Moreover, as stated by Ojiambo et al.[22], large differences exist in PA prevalence according to the choice of cut off points.

Consistent with previous studies [37, 38] , boys were more active than girls. This extra activity of our male CG could partly explain results of previous DXA studies [11] as male SWI presented lower BMD values at several sites when compared to CG. This does not make swimming negative per se, but while some adolescents are swimming, others might be doing other activities that might improve bone health. When focusing on the females a clear idea of how swimming affects bone is obtained as no differences were noticed between MVPA or VPA between female SWI and CG and no differences were found between bone variables. Therefore, this reinforces the idea that swimming may be neutral to bone mass, and that some of the effects found in previous studies could partly be explained by extra activity of the CG [11].

The present study is not exempt from limitations, as it is possible that other unmeasured factors could have contributed to bone mass, such as genetics or diet. In addition, accelerometers only register accelerations and therefore when performing an activity with extra weight the accelerometer will underestimate the total intensity of the activity. This could be an important limitation for future studies that try to replicate the present one in adult swimmers as adult swimmers unlike the sample of the present study perform a high amount of weight-lifting. In addition, sample size, especially in the female group might have masked some significant associations. It would therefore be interesting to perform future studies with a larger sample size in order to confirm this hypothesis. Nevertheless, accelerometry is an 
objective measure and has been reported to be one of the best measures for reporting PA[39]. Moreover, this is the first study to report objective PA in adolescent SWI and compare it to CG.

In conclusion, male SWI spent less time in weight-bearing VPA and MVPA than CG, which could partly explain lower BMD values in SWI than CG. Female SWI did not present any differences regarding PA levels or bone mass when compared to female CG, suggesting that swimming is neutral to bone mass. This is concerning, as childhood and adolescence are key periods for attaining peak bone mass. Weightbearing activities positively influenced bone mass in SWI as those that performed more than 60 minutes of MVPA per day showed higher bone health than those not complying with the recommended guidelines[23]. Randomized control trials including weight-bearing or exercise interventions should be performed on these athletes, in order to evaluate its effect on BMD and bone strength values. 


\section{REFERENCES}

1. $\quad$ Seeman E (2002) An exercise in geometry. J Bone Miner Res 17:373-380.

2. Bliuc D, Nguyen, ND, Nguyen, TV, Eisman, JA, Center, JR (2013) Compound risk of high mortality following osteoporotic fracture and refracture in elderly women and men. J Bone Miner Res 28:2317-2324.

3. Dent C (1972) Keynote address: problems in metabolic bone disease. Detroit: Henry Ford Hospital.

4. $\quad$ Bailey DA, McKay, HA, Mirwald, RL, Crocker, PR, Faulkner, RA (1999) A six-year longitudinal study of the relationship of physical activity to bone mineral accrual in growing children: the university of Saskatchewan bone mineral accrual study. J Bone Miner Res 14:1672-1679.

5. Brundtland GH (2001) Statement by Dr Gro Harlem Brundtland, Director General WHO, to the Fifth Global Conference on Health Promotion, Mexico City, 5 June 2000. Health Promot Int 16:95-98.

6. Vicente-Rodriguez G (2006) How does exercise affect bone development during growth? Sports Med 36:561-569.

7. Guadalupe-Grau A, Fuentes, T, Guerra, B, Calbet, JAL (2009) Exercise and bone mass in adults. Sports Medicine 39:439-468.

8. Olmedillas H, Gonzalez-Aguero, A, Moreno, LA, Casajus, JA, Vicente-Rodriguez, G (2012) Cycling and bone health: a systematic review. BMC Med 10:168.

9. Gomez-Bruton A, Gonzalez-Aguero, A, Gomez-Cabello, A, Casajus, JA, Vicente-Rodriguez, G (2013) Is bone tissue really affected by swimming? A systematic review. PLoS One 8:e70119.

10. Gomez-Bruton A, Montero-Marín, J, González-Agüero, A, García-Campayo, J, Moreno, L, Casajús, J, Vicente-Rodríguez, G (In press) The effect of swimming during adolescence on bone mineral density: A systematic review and meta-analysis. Sports Med.

11. Gomez-Bruton A, González-Agüero, A, Gómez-Cabello, A, Matute-Llorente, A, Casajús, J, Vicente-Rodríguez, G (2014) The effects of swimming training on bone tissue in adolescence. Scand J Med Sci Sports Online Version of Record published before inclusion in an issue.

12. Gomez-Bruton A, González-Agüero, A, Gómez-Cabello, A, Matute-Llorente, A, Zemel, B, Moreno, L, Casajús, J, Vicente-Rodríguez, G (In press) Bone structure of adolescent swimmers; a peripheral quantitative computed tomography (pQCT) study. Journal of Science and Medicine in Sport. 13. Heaney RP, Abrams, S, Dawson-Hughes, B, Looker, A, Marcus, R, Matkovic, V, Weaver, C (2000) Peak bone mass. Osteoporos Int 11:985-1009.

14. Hawkey A (2003) The importance of exercising in space. Interdiscip Sci Rev 28:130-138. 15. Gomez-Bruton A, González-Agüero, A, Casajus, JA, Vicente-Rodríguez, G (2014) Swimming training repercussion on metabolic and structural bone development. Benefits of the incorporation of whole body vibration or pliometric training. The RENACIMIENTO project. Nutr Hosp 30:399-409. 16. Tanner JM, Whitehouse, RH, Takaishi, M (1966) Standards from birth to maturity for height, weight, height velocity, and weight velocity: British children, 1965. II. Arch Dis Child 41:613-635. 17. Leone M, Comtois, AS (2007) Validity and reliability of self-assessment of sexual maturity in elite adolescent athletes. J Sports Med Phys Fitness 47:361-365.

18. Gonzalez-Aguero A, Vicente-Rodriguez, G, Gomez-Cabello, A, Casajus, JA (2013) Cortical and trabecular bone at the radius and tibia in male and female adolescents with Down syndrome: a peripheral quantitative computed tomography (pQCT) study. Osteoporos Int 24:1035-1044.

19. Pfeiffer KA, McIver, KL, Dowda, M, Almeida, MJ, Pate, RR (2006) Validation and calibration of the Actical accelerometer in preschool children. Med Sci Sports Exerc 38:152-157.

20. Treuth MS, Sherwood, NE, Butte, NF, McClanahan, B, Obarzanek, E, Zhou, A, Ayers, C, Adolph, A, Jordan, J, Jacobs, DR, Rochon, J (2003) Validity and reliability of activity measures in African-American girls for GEMS. Med Sci Sports Exerc 35:532-539.

21. Vanhelst J, Beghin, L, Turck, D, Gottrand, F (2011) New validated thresholds for various intensities of physical activity in adolescents using the Actigraph accelerometer. Int J Rehabil Res 34:175-177.

22. Ojiambo R, Cuthill, R, Budd, H, Konstabel, K, Casajus, JA, Gonzalez-Aguero, A, Anjila, E, Reilly, JJ, Easton, C, Pitsiladis, YP (2011) Impact of methodological decisions on accelerometer outcome variables in young children. Int J Obes (Lond) 35 Suppl 1:S98-103.

23. World Health Organization (2010) Global recommendations on physical activity for health. In. 24. Gracia-Marco L, Rey-Lopez, JP, Santaliestra-Pasias, AM, Jimenez-Pavon, D, Diaz, LE, Moreno, LA, Vicente-Rodriguez, G (2012) Sedentary behaviours and its association with bone mass in adolescents: the HELENA Cross-Sectional Study. BMC Public Health 12:971. 
25. Chastin SF, Mandrichenko, O, Helbostadt, JL, Skelton, DA (2014) Associations between objectively-measured sedentary behaviour and physical activity with bone mineral density in adults and older adults, the NHANES study. Bone 64:254-262.

26. Magkos F, Yannakoulia, M, Kavouras, SA, Sidossis, LS (2007) The type and intensity of exercise have independent and additive effects on bone mineral density. Int J Sports Med 28:773-779.

27. Gruodyte R, Jurimae, J, Cicchella, A, Stefanelli, C, Passariello, C, Jurimae, T (2010) Adipocytokines and bone mineral density in adolescent female athletes. Acta Paediatr 99:1879-1884.

28. Taaffe DR, Snow-Harter, C, Connolly, DA, Robinson, TL, Brown, MD, Marcus, R (1995)

Differential effects of swimming versus weight-bearing activity on bone mineral status of eumenorrheic athletes. J Bone Miner Res 10:586-593.

29. Janz KF, Letuchy, EM, Francis, SL, Metcalf, KM, Burns, TL, Levy, SM (2014) Objectively measured physical activity predicts hip and spine bone mineral content in children and adolescents ages 5-15 years: Iowa bone development study. Front Endocrinol (Lausanne) 5:112.

30. Sayers A, Mattocks, C, Deere, K, Ness, A, Riddoch, C, Tobias, JH (2011) Habitual levels of vigorous, but not moderate or light, physical activity is positively related to cortical bone mass in adolescents. J Clin Endocrinol Metab 96:E793-802.

31. Gracia-Marco L, Moreno, LA, Ortega, FB, Leon, F, Sioen, I, Kafatos, A, Martinez-Gomez, D, Widhalm, K, Castillo, MJ, Vicente-Rodriguez, G (2011) Levels of physical activity that predict optimal bone mass in adolescents: the HELENA study. Am J Prev Med 40:599-607.

32. Frost HM (2003) Bone's mechanostat: a 2003 update. Anat Rec A Discov Mol Cell Evol Biol 275:1081-1101.

33. Skerry TM (2006) One mechanostat or many? Modifications of the site-specific response of bone to mechanical loading by nature and nurture. J Musculoskelet Neuronal Interact 6:122-127.

34. Janssen I, Leblanc, AG (2010) Systematic review of the health benefits of physical activity and fitness in school-aged children and youth. Int J Behav Nutr Phys Act 7:40.

35. Gomez-Bruton A, González-Agüero, A, Gómez-Cabello, A, Matute-Llorente, A, Zemel, B, Moreno, L, Casajús, J, Vicente-Rodríguez, G (Under-review) Bone structure of adolescent swimmers; a peripheral quantitative computed tomography (pQCT) study. Journal of Science and Medicine in Sport. 36. Ruiz JR, Ortega, FB, Martinez-Gomez, D, Labayen, I, Moreno, LA, De Bourdeaudhuij, I, Manios, Y, Gonzalez-Gross, M, Mauro, B, Molnar, D, Widhalm, K, Marcos, A, Beghin, L, Castillo, MJ, Sjostrom, M (2011) Objectively measured physical activity and sedentary time in European adolescents: the HELENA study. Am J Epidemiol 174:173-184.

37. Trost SG, Pate, RR, Sallis, JF, Freedson, PS, Taylor, WC, Dowda, M, Sirard, J (2002) Age and gender differences in objectively measured physical activity in youth. Med Sci Sports Exerc 34:350-355. 38. Ekelund U, Sardinha, LB, Anderssen, SA, Harro, M, Franks, PW, Brage, S, Cooper, AR, Andersen, LB, Riddoch, C, Froberg, K (2004) Associations between objectively assessed physical activity and indicators of body fatness in 9- to 10-y-old European children: a population-based study from 4 distinct regions in Europe (the European Youth Heart Study). Am J Clin Nutr 80:584-590.

39. Sirard JR, Pate, RR (2001) Physical activity assessment in children and adolescents. Sports Med 31:439-454. 
Figure 1. Age, lean and height or object length adjusted differences in bone mass measured by DXA and PQCT between male active and inactive swimmers.

$*=p<0.05$ 